\title{
Locally Defined Operators in the Space of Functions of Bounded Riesz-Variation
}

\author{
W. Aziz, ${ }^{1}$ J. A. Guerrero, ${ }^{2}$ K. Maldonado, ${ }^{2}$ and N. Merentes $^{3}$ \\ ${ }^{1}$ Universidad de Los Andes, Departamento de Física y Matemática, Trujillo, Venezuela \\ ${ }^{2}$ Universidad Nacional Experimental del Táchira, Departamento de Matemática y Física, San Cristóbal, Venezuela \\ ${ }^{3}$ Universidad Central de Venezuela, Escuela de Matemáticas, Caracas, Venezuela \\ Correspondence should be addressed to W. Aziz; wadie@ula.ve
}

Received 14 October 2014; Revised 11 January 2015; Accepted 16 January 2015

Academic Editor: Abdellatif Agouzal

Copyright (C) 2015 W. Aziz et al. This is an open access article distributed under the Creative Commons Attribution License, which permits unrestricted use, distribution, and reproduction in any medium, provided the original work is properly cited.

We study the locally defined operator on the spaces of bounded Riesz p-variation functions and we prove that those operators are the Nemytskii operator.

\section{Introduction}

We have an closed interval $I$ of the real line and let $\mathscr{X}=$ $\mathscr{X}(I), \mathscr{Y}=\mathscr{Y}(I)$ be function spaces $f: I \rightarrow \mathbb{R}$. An operator $K: \mathscr{X} \rightarrow \mathscr{Y}$ is called a locally defined operator, or $(X, \mathscr{Y})$ local operator, briefly, a local operator [1], if for every open interval $J \subset \mathbb{R}$ and for all functions $f, g \in \mathscr{X}$, the implication

$$
\left.f\right|_{J \cap I}=\left.\left.g\right|_{J \cap I} \Longrightarrow K(f)\right|_{J \cap I}=\left.K(g)\right|_{J \cap I}
$$

is true, where $\left.f\right|_{J \cap I}$ denotes the restriction of $f$ to $J \cap I$.

There is a vast literature on the problem treated here, mainly compiled of definitions of locally defined operators involving a measure space (cf., e.g., [2-5]). Also we proved that, in general, $K$ is a composition (or Nemytskii) operator of the form $K(f)(x)=h(x, f(x))$ for a two-variable function $h$. Assuming additionally that $K$ is continuous in measure, the generating function $h$ can be replaced by a function satisfying the Caratheodory conditions (cf. [6]). The present paper concerns topological aspects of locally defined operators (cf. [1,7-10]). For more knowledge on theory of the composition operators, see Appell and Zabrejko [11]. In [7] it was done is the case when $\mathscr{X}=C^{n}(I)$ and $\mathscr{Y}=C(I)$ or $y=C^{1}(I)$. Subsequently, this result has been extended by several authors: $[8,9,12]$ (for spaces of Whitney differentiable functions), [10, 13] (for space of Hölder functions), [14] (for continuous and monotone functions), and [1] (for functions of bounded $\varphi$-variation in the sense Wiener). In the present paper we are interested in such operators in the context of bounded Riesz-variation functions. In particular, we show that if the operator $K$ maps the space $R V_{p}(I, \mathbb{R})$ into itself and is locally defined, then $K$ is a Nemytskii composition operator.

\section{Notation and Preliminaries}

In this section we present some necessary notations and definitions and recall some knowledge concerning the bounded Riesz-variation.

In the sequel, $\mathbb{N}, \mathbb{N}_{0}$, and $\mathbb{R}$ denote, respectively, the set of positive integers, nonnegative integers, and the set of real numbers.

Let $I=[a, b] \subset \mathbb{R} ; \pi=\left\{t_{i}\right\}_{i=0}^{m}$ be partition of $I$, defined by $\pi: a=t_{0}<t_{1}<\cdots<t_{m}=b$. As usually, $\mathbb{R}^{I}$ denote the family of all functions $f: I \rightarrow \mathbb{R}$.

Given $1 \leq p<\infty, f \in \mathbb{R}^{I}$ and a partition $\pi$ of $I$, we define

$$
v_{p}^{R}(f)=v_{p}^{R}(f, I):=\sup _{\pi} \sum_{i=1}^{m} \frac{\left|f\left(t_{i}\right)-f\left(t_{i-1}\right)\right|^{p}}{\left|t_{i}-t_{i-1}\right|^{p-1}},
$$

where the supremum is taken over all partitions $\pi$ of $I . v_{p}^{R}(f)$ is the classical $p$-variation of $f$ in the sense of Riesz [15] in $I$. A function $f$ is said to be of bounded $p$-variation if 
$v_{p}^{R}(f, I)<\infty$. By $R V_{p}(I)$ we denote the Banach space of all functions $f \in \mathbb{R}^{I}$ of bounded $p$-variation equipped with the norm

$$
\|f\|_{p}:=|f(a)|+\left(v_{p}^{R}(f)\right)^{1 / p}, \quad f \in R V_{p}(I) .
$$

Lemma 1. Let $I=[a, b] \subset \mathbb{R}$ be an interval and let $\left(x_{0}, y_{0}\right) \in$ $I \times \mathbb{R}, x_{0}<\sup (I)$ be fixed. Then for every sequence $\left(x_{k}, y_{k}\right) \in$ $I \times \mathbb{R}$ satisfying the condition

$$
\lim _{k \rightarrow \infty}\left(x_{k}, y_{k}\right)=\left(x_{0}, y_{0}\right) \text {, }
$$

$$
x_{k+1}<x_{k} ; y_{k+1}<y_{k}, k \in \mathbb{N}
$$

there exists a function $\psi \in R V_{p}(I)$ such that, for all $k \in \mathbb{N}_{0}$,

$$
\psi\left(x_{k}\right)=y_{k} .
$$

Proof. Take an arbitrary sequence $\left(x_{k}, y_{k}\right) \in I \times \mathbb{R}$ satisfying (4) and define a sequence of functions $\psi_{k}: I \rightarrow \mathbb{R}, k \in \mathbb{N}$, by

$$
\begin{aligned}
& \psi_{k}(x) \\
& := \begin{cases}y_{0}, & \text { for } x \in\left[a, x_{0}\right] ; \\
\frac{y_{k}-y_{0}}{x_{k}-x_{0}}\left(x-x_{0}\right)+y_{0}, & \text { for } x \in\left(x_{0}, x_{k}\right] ; \\
\frac{y_{i}-y_{i-1}}{x_{i}-x_{i-1}}\left(x-x_{i}\right)+y_{i}, & \text { for } x \in\left(x_{i}, x_{i-1}\right], i \in\{2, \ldots, k\} ; \\
y_{1}, & \text { for } x \in\left(x_{1}, b\right] .\end{cases}
\end{aligned}
$$

Let us observe that

$$
\begin{aligned}
& \psi_{k}\left(x_{0}\right)=y_{0}, \\
& \psi_{k}\left(x_{k}\right)=\psi_{k+\ell}\left(x_{k}\right)=y_{k},
\end{aligned}
$$

$k, \ell \in \mathbb{N}$

and for every $x \in I \backslash\left\{x_{k}: k \in \mathbb{N}_{0}\right\}$ there exist $k_{0} \in \mathbb{N}$ such that

$$
\psi_{k}(x)=\psi_{k_{0}}(x), \quad k \geq k_{0}, k \in \mathbb{N} .
$$

Put

$$
\psi(x)=\lim _{k \rightarrow \infty} \psi_{k}(x), \quad x \in I .
$$

From (7) and (8), the function $\psi$ is well defined. Moreover, $\psi$ is nondecreasing and

$$
\psi\left(x_{k}\right)=y_{k}, \quad \forall k \in \mathbb{N}_{0},
$$

and by (9), for each $\epsilon>0$, we obtain

$$
\left|\psi_{k}(x)-\psi(x)\right|<\epsilon, \quad \forall x \in I,
$$

so $\left\|\psi_{k}-\psi\right\|_{\infty} \leq \epsilon$. Thus the sequence $\left(\psi_{k}\right)_{k \in \mathbb{N}}$ tends uniformly to $\psi$.

Now as $\psi_{k} \in R V_{p}(I)$ for all $k \in \mathbb{N}$ and $\psi_{k}$ tends uniformly to $\psi$, then

$$
\begin{aligned}
\frac{\left|\psi\left(x_{i}\right)-\psi\left(x_{i-1}\right)\right|^{p}}{\left|x_{i}-x_{i-1}\right|^{p-1}} & =\lim _{k \rightarrow \infty} \frac{\left|\psi_{k}\left(x_{i}\right)-\psi_{k}\left(x_{i-1}\right)\right|^{p}}{\left|x_{i}-x_{i-1}\right|^{p-1}} \\
& \leq \lim _{k \rightarrow \infty} v_{p}^{R}\left(\psi_{k}, I\right)<\infty ;
\end{aligned}
$$

thus $v_{p}^{R}(\psi, I)<\infty$ and therefore $\psi \in R V_{p}(I)$.

Similarly, we can get the following.
Remark 2. If $\left(x_{0}, y_{0}\right) \in I \times \mathbb{R}$, where $x_{0}>\inf (I)$ and $\left(x_{k}, y_{k}\right) \in$ $I \times \mathbb{R}$ is a sequence satisfying the condition

$$
\lim _{k \rightarrow \infty}\left(x_{k}, y_{k}\right)=\left(x_{0}, y_{0}\right) \text {, }
$$

$$
x_{k}<x_{k+1} ; y_{k} \leq y_{k+1}, k \in \mathbb{N},
$$

then there exists a function $\psi \in R V_{p}(I)$ such that, for all $k \in$ $\mathbb{N}_{0}$,

$$
\psi\left(x_{k}\right)=y_{k}
$$

\section{Locally Defined Operators}

Now we can introduce the definition of the local defined operators of type $K: R V_{p}(I) \rightarrow C(I)$.

Definition 3 (see [1]). An operator $K: R V_{p}(I) \rightarrow C(I)$ is said to be locally defined, if, for every two functions $f, g \in R V_{p}(I)$ and for every open interval $J \subset \mathbb{R}$,

$$
\left.f\right|_{J \cap I}=\left.\left.g\right|_{J \cap I} \Longrightarrow K(f)\right|_{J \cap I}=\left.K(g)\right|_{J \cap I} .
$$

Theorem 4. Let $1<p<\infty$. If a locally defined operator $K$ maps $R V_{p}(I)$ into $C(I)$, then there exists a unique function $h$ : $I \times \mathbb{R} \rightarrow \mathbb{R}$ such that, for all $f \in R V_{p}(I)$,

$$
K(f)(t)=h(t, f(t)), \quad t \in I .
$$

Proof. We begin by showing that, for every $f, g \in R V_{p}(I)$ and, for every $x_{0} \in \operatorname{int}(I)$, the condition

$$
f\left(x_{0}\right)=g\left(x_{0}\right)
$$

implies that

$$
K(f)\left(x_{0}\right)=K(g)\left(x_{0}\right) .
$$

To this end choose arbitrary $x_{0} \in \operatorname{int}(I)$ and take an arbitrary pair of functions $f, g \in R V_{p}(I)$ which fulfil (17) (i.e., $f\left(x_{0}\right)=$ $\left.g\left(x_{0}\right)\right)$. The function $\varphi: I \rightarrow \mathbb{R}$, defined by

$$
\varphi(x)= \begin{cases}f(x), & \text { for } x \in\left[a, x_{0}\right] ; \\ g(x), & \text { for } x \in\left(x_{0}, b\right],\end{cases}
$$

belongs to $R V_{p}(I)$. Indeed, define the functions $f_{1}, g_{1}: I \rightarrow$ $\mathbb{R}$ by

$$
\begin{aligned}
& f_{1}(x)= \begin{cases}f(x)-f\left(x_{0}\right), & \text { for } x \in\left[a, x_{0}\right] ; \\
0, & \text { for } x \in\left(x_{0}, b\right],\end{cases} \\
& g_{1}(x)= \begin{cases}0, & \text { for } x \in\left[a, x_{0}\right] ; \\
g(x)-g\left(x_{0}\right), & \text { for } x \in\left(x_{0}, b\right]\end{cases}
\end{aligned}
$$

Since $f, g \in R V_{p}(I), v_{p}^{R}(f)<\infty$ and $v_{p}^{R}(g)<\infty$. Let $\pi=\left\{x_{i}\right\}_{i=0}^{m}$ be a partition of $I$ such that $x_{\ell-1} \leq x_{0}<x_{\ell}$ for some $1 \leq \ell \leq m$. Then

$$
\sum_{i=1}^{m} \frac{\left|f_{1}\left(x_{i}\right)-f_{1}\left(x_{i-1}\right)\right|^{p}}{\left|x_{i}-x_{i-1}\right|^{p-1}} \leq v_{p}^{R}(f) .
$$


Hence $v_{p}^{R}\left(f_{1}\right)<\infty$. By a similar reasoning, we have $v_{p}^{R}\left(g_{1}\right)<$ $\infty$. Finally $f_{1}+g_{1} \in R V_{p}(I)$, as $R V_{p}(I)$ is a linear space. Thus

$$
v_{p}^{R}\left(f_{1}+g_{1}\right)<\infty \text {. }
$$

Since, for all $x, x^{\prime} \in I$

$$
\left(f_{1}+g_{1}\right)(x)-\left(f_{1}+g_{1}\right)\left(x^{\prime}\right)=\psi(x)-\psi\left(x^{\prime}\right),
$$

the condition (22) implies that $\varphi \in R V_{p}(I)$.

As

$$
\begin{gathered}
\left.f\right|_{\left(-\infty, x_{0}\right) \cap I}=\left.\varphi\right|_{\left(-\infty, x_{0}\right) \cap I}, \\
\left.g\right|_{\left(x_{0}, \infty\right) \cap I}=\left.\psi\right|_{\left(x_{0}, \infty\right) \cap I},
\end{gathered}
$$

by definition of a local operator, we get

$$
\begin{gathered}
\left.K(f)\right|_{\left(-\infty, x_{0}\right) \cap I}=\left.K(\varphi)\right|_{\left(-\infty, x_{0}\right) \cap I}, \\
K(g)_{\left(x_{0}, \infty\right) \cap I}=K(\varphi)_{\left(x_{0}, \infty\right) \cap I} .
\end{gathered}
$$

Therefore, by the continuity of $K(f), K(g)$ and $K(\varphi)$ at $x_{0}$, we obtain

$$
K(f)\left(x_{0}\right)=K(\varphi)\left(x_{0}\right)=K(g)\left(x_{0}\right) .
$$

Suppose now that $x_{0}$ is the left endpoint of the interval $I$ (i.e., $\left.x_{0}=a\right)$. There exists a sequence $\left(x_{k}, y_{k}\right) \in I \times \mathbb{R}$ such that $x_{0}<x_{k+1}<x_{k}, y_{0} \leq y_{k+1}<y_{k}, k \in \mathbb{N}$, and by the continuity of $f$ and $g$ at $x_{0}$

$$
\lim _{k \rightarrow \infty}\left(x_{k}, y_{k}\right)=\left(x_{0}, y_{0}\right)
$$

By Lemma 1 there exists a function $\psi \in R V_{p}(I)$ such that $\psi\left(x_{k}\right)=y_{k}$ for all $k \in \mathbb{N}_{0}$.

There is no loss of generality in supposing that $f\left(x_{0}\right)=$ $g\left(x_{0}\right)=y_{0}, \psi\left(x_{2 k-1}\right)=y_{2 k-1}=g\left(x_{2 k-1}\right)$ and $\psi\left(x_{2 k}\right)=y_{2 k}=$ $f\left(x_{2 k}\right), k \in \mathbb{N}$.

According to the first part of the proof, we have

$$
\begin{gathered}
K(\psi)\left(x_{2 k-1}\right)=K(g)\left(x_{2 k-1}\right), \\
K(\psi)\left(x_{2 k}\right)=K(f)\left(x_{2 k}\right),
\end{gathered}
$$

\section{$k \in \mathbb{N}$.}

Hence, by continuity of $K(\psi), K(g)$, and $K(f)$ at $x_{0}$, letting $k \rightarrow \infty$, we get

$$
K(f)\left(x_{0}\right)=K(\psi)\left(x_{0}\right)=K(g)\left(x_{0}\right) .
$$

When $x_{0}$ is the right endpoint of $I$, the argument is similar.

To define the function $h: I \times \mathbb{R} \rightarrow \mathbb{R}$ and fix arbitrarily an $y_{0} \in \mathbb{R}$, let us define a function $P_{y_{0}}: I \rightarrow \mathbb{R}$ by

$$
P_{y_{0}}(x):=y_{0}, \quad x \in I .
$$

Of course $P_{y_{0}}$, as a constant function, belongs to $R V_{p}(I)$. For $x_{0} \in I, y_{0} \in \mathbb{R}$, put

$$
h\left(x_{0}, y_{0}\right):=K\left(P_{y_{0}}\right)\left(x_{0}\right) \text {. }
$$

Since, by (30), for all functions $f$,

$$
f\left(x_{0}\right)=P_{f\left(x_{0}\right)}\left(x_{0}\right),
$$

according to what has already been proved, we have

$$
K(f)\left(x_{0}\right)=K\left(P_{f\left(x_{0}\right)}\right)\left(x_{0}\right)=h\left(x_{0}, f\left(x_{0}\right)\right) .
$$

To prove the uniqueness of $h$, assume that $\bar{h}: I \times \mathbb{R} \rightarrow \mathbb{R}$ is such that

$$
K(f)(x)=\bar{h}(x, f(x))
$$

for all $f \in R V_{p}(I)$ and $x \in I$. To show that $h=\bar{h}$ let us fix arbitrarily $x \in I, y \in \mathbb{R}$ and take $f \in R V_{p}(I)$ with $f(x)=y$. From (33), we have

$$
\begin{aligned}
\bar{h}(x, y) & =\bar{h}(x, f(x))=K(f)(x)=h(x, f(x)) \\
& =h(x, y)
\end{aligned}
$$

which proves the uniqueness of $h$.

Definition 5. Let $X \subset \mathbb{R}$ and a function $h: X \times \mathbb{R} \rightarrow \mathbb{R}$ be fixed. The mapping $H: \mathbb{R}^{X} \rightarrow \mathbb{R}^{X}$, given by

$$
H(f)(x):=h(x, f(x)), \quad f \in \mathbb{R}^{X}, x \in X,
$$

is said to be composition (Nemytskii or superposition) operator. The function $h$ is referred to as the generator of the operator $H$.

As an immediate consequence of Theorem 4 we get the following.

Corollary 6. Let $1 \leq p<\infty$. If a local operator $K$ maps $R V_{p}(I)$ into $C(I)$, then it is a Nemytskii operator.

Note that if a local operator $K$ maps $R V_{p}(I)$ into itself then, obviously, $K$ maps $R V_{p}(I)$ into $C(I)$. Therefore, by Theorem 4, we get the following.

Theorem 7. Let $1 \leq p<\infty$. If a local operator $K$ maps $R V_{p}(I)$ into itself, then there exists a unique function $h: I \times \mathbb{R} \rightarrow \mathbb{R}$ such that, for all $f \in R V_{p}(I)$,

$$
K(f)(x)=h(x, f(x)), \quad x \in I .
$$

Corollary 8. Let $1 \leq p<\infty$. If a local operator $K$ maps $R V_{p}(I)$ into itself, then it is a Nemytskii operator.

Under the additional assumption that the locally defined operator is uniformly continuous, we get a complete characterization of its generating function $h$. Namely, we have the following.

Theorem 9. Let $1 \leq p<\infty$. If a local operator $K$ : $R V_{p}(I) \rightarrow R V_{p}(I)$ is uniformly continuous, then there exists $f_{1}, f_{2} \in R V_{p}(I)$ such that

$$
K(f)(x)=f_{1}(x) f(x)+f_{2}(x),
$$

$f \in R V_{p}(I), x \in I$. 
Proof. From Theorem 7 there exists a unique function $h: I \times$ $\mathbb{R} \rightarrow \mathbb{R}$ such that $K(f)(x)=h(x, f(x))$ for all $f \in R V_{p}(I)$, $x \in I$. Fix $\left(x_{0}, y_{0}\right) \in I \times \mathbb{R}$, take an arbitrary sequence $x_{n} \in I$ with $x_{n} \rightarrow x_{0}$, and let $P_{y_{0}}: I \rightarrow \mathbb{R}$ be defined by $P_{y_{0}}(x)=$ $y_{0}, x \in I$. Since $h\left(x_{0}, y_{0}\right)=K\left(P_{y_{0}}\right)\left(x_{0}\right)$,

$$
\begin{aligned}
\mid h & \left(x_{n}, y_{0}\right)-h\left(x_{0}, y_{0}\right) \mid \\
& =\left|h\left(x_{n}, P_{y_{0}}\left(x_{n}\right)\right)-h\left(x_{0}, P_{y_{0}}\left(x_{0}\right)\right)\right| \\
& =\left|K\left(P_{y_{0}}\right)\left(x_{n}\right)-K\left(P_{y_{0}}\right)\left(x_{0}\right)\right| ;
\end{aligned}
$$

applying the continuity of $K\left(P_{y_{0}}\right)$ at $x_{0}$, we get the continuity of $h$ with respect to the first variable. Thus, by [16, Theorem 1] (with $\left.\varphi(x)=\psi(x)=x^{p}\right)$,

$$
h(x, y)=f_{1}(x) y+f_{2}(x), \quad x \in I, y \in \mathbb{R},
$$

for some $f_{1}, f_{2}: I \rightarrow \mathbb{R}$. Since $h\left(\cdot, y_{0}\right)=K\left(P_{y_{0}}\right)(\cdot) \in R V_{p}(I)$ and $f_{2}(x)=h(x, 0), f_{1}(x)=h(x, 1)-f_{2}(x)$, the functions $f_{1}, f_{2} \in R V_{p}(I)$.

\section{Conflict of Interests}

The authors declare that there is no conflict of interests regarding the publication of this paper.

\section{Acknowledgments}

The authors are grateful to the referee for his valuable comments and suggestions. W. Aziz wants to mention that this research was partly supported by CDCHTA of Universidad de Los Andes under the project NURR-C-584-15-05B. J. A. Guerrero and K. Maldonado were partially funded by the Decanato de Investigación, Universidad Nacional Experimental del Táchira-Venezuela, under the Project 04011-2015. This research has been partly supported by Central Bank of Venezuela.

\section{References}

[1] M. Wrobel, "Locally defined operators in the space of functions of bounded $\varphi$-variation," Real Analysis Exchange, vol. 38, no. 1, pp. 79-94, 2013.

[2] K. Karták, "A generalization of the Carathéodory theory of differential equations," Czechoslovak Mathematical Journal, vol. 17, pp. 482-514, 1967.

[3] K. Karták, "On Carathéodory operators," Czechoslovak Mathematical Journal, vol. 17, no. 92, pp. 515-519, 1967.

[4] W. Kozlowski, "Nonlinear opeartors in funtion Banach spaces," Commentationes Mathematicae. Prace Matematyczne, vol. 22, pp. 85-103, 1980.

[5] I. V. Shragin, "On representation of a locally defined operator in the form of the Nemytskii operator," Functional Differential Equations, vol. 3, no. 3-4, pp. 447-452, 1996.

[6] J. Appell and P. P. Zabreiko, "Continuity properties of the superposition operators," Journal of the Australian Mathematical Society. Series A, vol. 47, no. 2, pp. 186-210, 1989.

[7] K. Lichawski, J. Matkowski, and J. Miś, "Locally defined operators in the space of differentiable functions," Bulletin of the Polish
Academy of Sciences. Mathematics, vol. 37, no. 1-6, pp. 315-325, 1989.

[8] J. Matkowski and M. Wróbel, "Locally defined operators in the space of Whitney differentiable functions," Nonlinear Analysis: Theory, Methods \& Applications, vol. 68, no. 10, pp. 2933-2942, 2008.

[9] J. Matkowski and M. Wrobel, "Representation theorem for locally defined operators in the space of Whitney differentiable functions," Manuscripta Mathematica, vol. 129, no. 4, pp. 437448, 2009.

[10] M. Wróbel, "Locally defined operators in the Hölder's spaces," Nonlinear Analysis. Theory, Methods \& Applications, vol. 74, no. 1, pp. 317-323, 2011.

[11] J. Appell and P. P. Zabrejko, Nonlinear Superposition Operators, Cambridge University Press, Cambridge, UK, 1990.

[12] M. Wróbel, "Locally defined operators and a partial solution of a conjecture," Nonlinear Analysis: Theory, Methods \& Applications, vol. 72, no. 1, pp. 495-506, 2010.

[13] J. Matkowski and M. Wrobel, The Bounded Local Operators in the Banach Space of Hölder Functions, vol. 15 of Scientific Issues, Mathematics, Jan Dlugosz University in Czestochowa, 2010.

[14] M. Wrobel, "Representation theorem for local operators in the space of continuous and monotone functions," Journal of Mathematical Analysis and Applications, vol. 372, no. 1, pp. 4554, 2010.

[15] F. Riesz, "Untersuchungen über Systeme integrierbarer Funktionen," Mathematische Annalen, vol. 69, no. 4, pp. 449-497, 1910.

[16] A. Acosta, W. Aziz, J. Matkowski, and N. Merentes, "Uniformly continuous composition operator in the space of $\varphi$-variation functions in the sense of Riesz," Fasciculi Mathematici, vol. 43, pp. 1-7, 2010. 


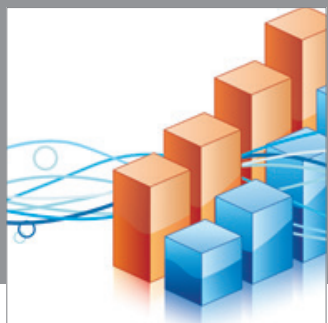

Advances in

Operations Research

mansans

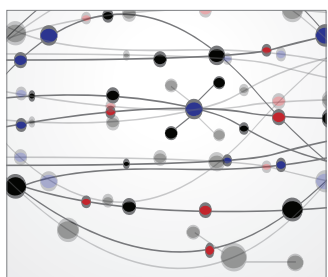

The Scientific World Journal
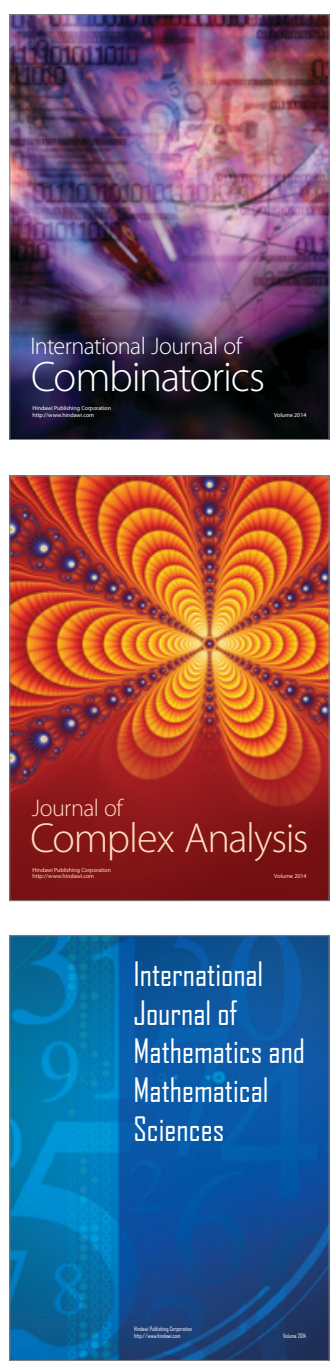
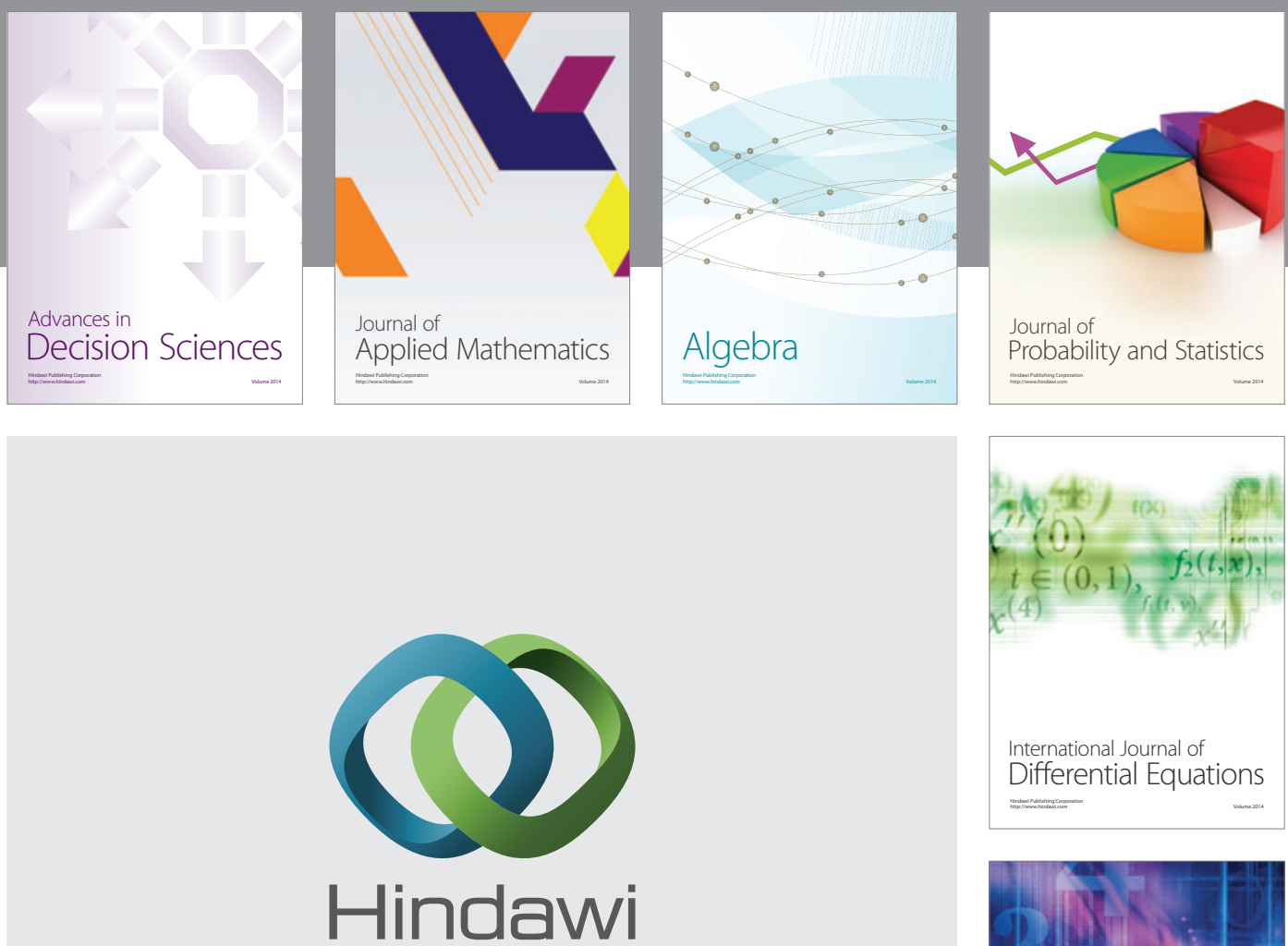

Submit your manuscripts at http://www.hindawi.com
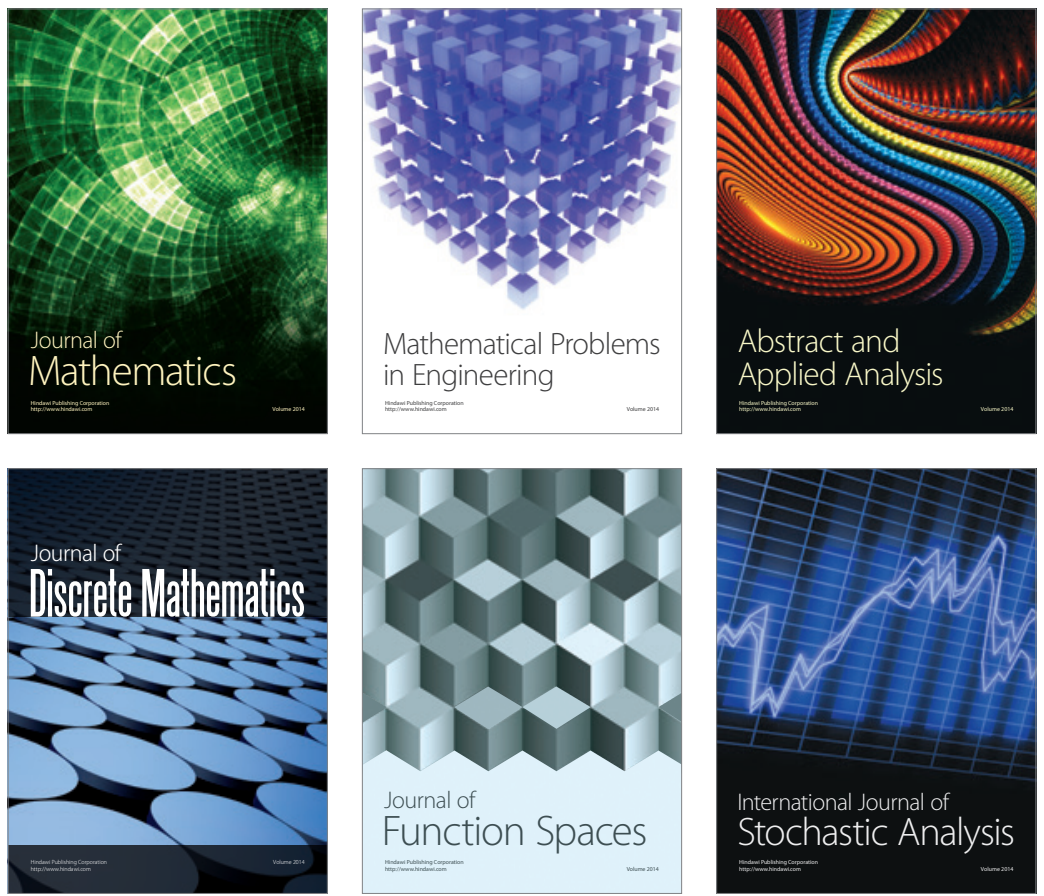

Journal of

Function Spaces

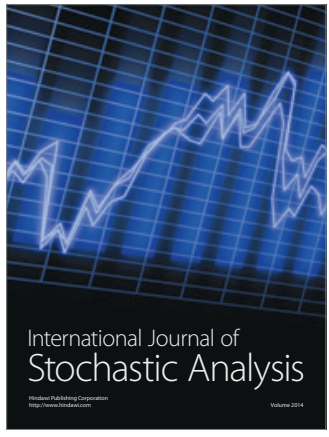

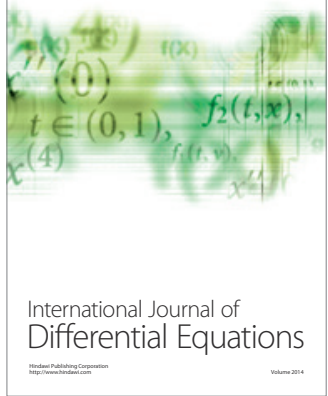
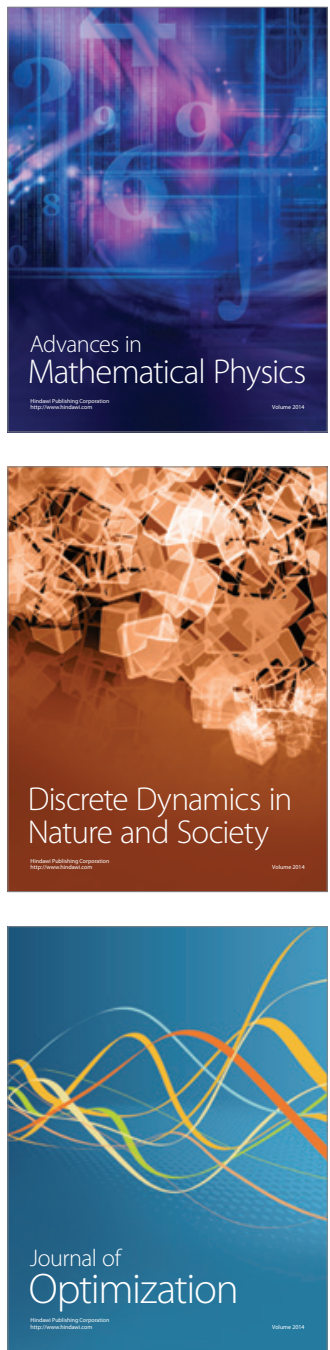